\title{
Starch-based hydrogels show relevant properties for tissue engineering and loading of nanoparticulate systems.
}

\author{
Seidy Pedroso-Santana ${ }^{1}$, Brian Rivas ${ }^{1}$, Noralvis Fleitas-Salazar ${ }^{1}$, Rafael Maura ${ }^{1}$, Carolina \\ Gomez-Gaete $^{1}$, Alexis Debut ${ }^{2}$, Natalie Parra ${ }^{1}$, Karla Vizuete ${ }^{2}$, Thelvia I. Ramos ${ }^{2}$, and \\ Jorge Toledo ${ }^{1}$ \\ ${ }^{1}$ Universidad de Concepcion \\ ${ }^{2}$ Universidad de las Fuerzas Armadas
}

May 11, 2020

\begin{abstract}
The synthesis of starch-based physical hydrogels in combination with chitosan and polyvinyl alcohol, and their potential coapplication with chitosan nanoparticles was evaluated. The potential of starch-chitosan hydrogel obtained by physical/chemical method for tissue engineering uses was also studied in a mouse wound healing model. Although the microscopical structure of each synthesized hydrogel suggests a possible biological application, starch-polyvinyl alcohol hydrogel exhibited rigid behavior with minor channel diameters, a lower swelling rate (less than 300\%), and negatively affected cell viability in a cytotoxicity assay. Starch-chitosan hydrogel obtained by chemical crosslinking with glutaraldehyde demonstrated the higher swelling rate (about $1100 \%$ ), cell viability values over $80 \%$, and a homogeneous tri-dimensional structure; along with an excellent interaction with chitosan nanoparticles. This type of hydrogel was selected for an in vivo experiment, showing significant differences in wound healing process against a non-treated control, in terms of inflammation, exudate production and tissue recovering.
\end{abstract}

\section{Starch-based hydrogels show relevant properties for tissue engineering and loading of nanopar- ticulate systems.}

Seidy Pedroso-Santana ${ }^{\mathrm{a}}$, Brian Ignacio Rivas Tiznado ${ }^{\mathrm{a}}$, Noralvis Fleitas-Salazar ${ }^{\mathrm{a}}$, Rafael Maura ${ }^{\mathrm{a}}$, Carolina Gómez-Gaete $^{\mathrm{b}}$, Alexis Debut ${ }^{\mathrm{c}}$, Natalie Parra ${ }^{\mathrm{a}}$, Karla S. Vizuete Armendariz ${ }^{\mathrm{c}}$, Thelvia I. Ramos ${ }^{\mathrm{c}}$, Jorge R. Toledo ${ }^{\mathrm{a}^{*}}$.

${ }^{a}$ Laboratorio de Biotecnología y Biofármacos, Departamento de Fisiopatología, Facultad de Ciencias Biológicas, Universidad de Concepción, Barrio Universitario s/n, Concepción CP. 4030000, Chile.

${ }^{\mathrm{b}}$ Departamento de Farmacia, Facultad de Farmacia, Universidad de Concepción, Barrio Universitario s/n, Concepción CP. 4030000, Chile.

${ }^{\mathrm{c}}$ Departamento Ciencias de la Vida y de la Agricultura, Universidad de las Fuerzas Armadas ESPE, Av. General Rumiñahui s/n, PO BOX 171-5-231B, Sangolqui, Ecuador.

*Corresponding author : Professor Jorge R. Toledo, jotoledo@udec.cl

\section{Abstract :}

The synthesis of starch-based physical hydrogels in combination with chitosan and polyvinyl alcohol, and their potential co-application with chitosan nanoparticles was evaluated. The potential of starch-chitosan hydrogel obtained by physical/chemical method for tissue engineering uses was also studied in a mouse wound healing model. Although the microscopical structure of each synthesized hydrogel suggests a possible biological application, starch-polyvinyl alcohol hydrogel exhibited rigid behavior with minor channel diameters, a lower 
swelling rate (less than 300\%), and negatively affected cell viability in a cytotoxicity assay. Starch-chitosan hydrogel obtained by chemical crosslinking with glutaraldehyde demonstrated the higher swelling rate (about $1100 \%$ ), cell viability values over $80 \%$, and a homogeneous tri-dimensional structure; along with an excellent interaction with chitosan nanoparticles. This type of hydrogel was selected for an in vivoexperiment, showing significant differences in wound healing process against a non-treated control, in terms of inflammation, exudate production and tissue recovering.

Keywords : hydrogel, starch, chitosan, nanoparticles loading, tissue engineering.

\section{Introduction .}

Hydrogels are polymeric three-dimensional structures, which absorb large quantities of aqueous solutions (Hamedi, 2018). This property is useful for drug delivery applications, based on retention and later release of hydrophilic drugs. Several polymers, such as chitosan (Huang, 2018), polyvinyl alcohol (Hou, 2015), starch (Biduski, 2018) and polyvinyl pyrrolidone (Timaeva, 2020), are currently used for hydrogels synthesis (Akhtar, 2016).

Starch is a natural polymer, obtained from plants, composed by repetitive units of amylose and amylopectin (Ismail, 2013). Its biocompatibility, biodegradability, high hydrophilicity, and low cost in the market makes this material an appropriate option in the production of hydrogels for biological applications (Perez, 2018). Starch-based hydrogels can be synthesized by copolymerization of polysaccharide monomers, using a chemical crosslinker, or under the action of physical initiators like temperature (Ismail, 2013). Combination of starch with other polymers is a way to obtain hydrogels with outstanding features in terms of structure strength, pore diameter in the hydrogel matrix, bio-adhesion, and response to environmental changes (Ou, 2017).

In general, hydrogel synthesis is carried out by physical or chemical methods, or by a combination of both (Ou, 2017). Physical hydrogels, also called self-assembling hydrogels (Fu, 2018), are relatively easy to obtain, based on non-covalent interactions between polymeric chains (ionic, electrical, etc.). One of the most used physical methods is freeze-thawing; by which the formation of microcrystals is responsible of the interaction among several polymeric planes, leading to a network-like tridimensional structure (Gulrez, 2011).

On the other hand, chemical methods frequently use cross-linking agents to link polymeric chains by covalent bonds (Gulrez, 2011). These interactions occur between polymer functional groups (-COOH, $-\mathrm{NH}_{2}$, etc.) and cross-linker groups. For example, glutaraldehyde (GA) is a chemical cross-linker widely known by interact with amines through its carbonyl (-CHO) groups (Crescenzi, 2003; Fadouloglou, 2008). This reaction is easy to obtain, but if there are proteins involved, its biological activity could be affected (Vrsanská, 2018).

The use of hydrogels in pharmaceutical applications provides drug protection and controlled release (LopezCordoba, 2019). Nevertheless, considering the small sizes of bioactive molecules in comparison to the size of hydrogel pores, the quantity of retained drug could be variable. One approach to obtain higher values of retention and an increased bioavailability is the formulation of active molecules inside nano and microparticles (Lopez-Cordoba, 2019; Perez, 2018). Polymeric nano/microparticles provide an additional protection to drugs along with a larger interaction surface, which allows increased drug retention inside hydrogel networks.

Here, we aimed to obtain different variants of starch-based hydrogels in combination (or not) with other biocompatible polymers: chitosan and polyvinyl alcohol. Physical and physical/chemical procedures were applied to synthesize hydrogel structures, which were later loaded with chitosan (CS) nanoparticles. Subsequently, in vitro and in vivo experiments corroborated the relevance of the systems developed in tissue engineering applications and for nanoparticulate systems loading.

Materials and methods .

Materials .

Low molecular weight deacetylated chitosan (50-190 KDa, 75-85\% deacetylated), sodium tri-polyphosphate (TPP), acetic acid, starch (soluble, ACS reagent), polyvinyl alcohol (PVA) (31-50 KDa. 98-99\% hydrolized), 
glutaraldehyde (GA) and all the reagents used for molecular biology and cell culture, were acquired from Sigma-Aldrich (USA). Nitrocellulose membranes of $0.45 \mu \mathrm{m}$ were from Merck (Germany).

Hydrogel synthesis .

Four variants of starch-based hydrogels were synthesized: 1) Starch, 2) Starch-PVA, 3) Starch-CS, 4) StarchCS-GA; similar to the procedures previously described (Liu, 2010; Bursali, 2011). Starch solution 8\% (w/v) and Starch - PVA solution 5\% - 5\% (w/v), in distilled water, were prepared by stirring at $500 \mathrm{rpm}$ with subsequent autoclavation (variants 1 and 2). The solutions were cooled at room temperature while stirred at $500 \mathrm{rpm}$. Variant 3 was prepared by dissolution of $5 \%$ starch and $5 \%$ CS (w/v) in acetic acid, $0.5 \%$ in water $(\mathrm{v} / \mathrm{v})$. The mixture was stirred at $800 \mathrm{rpm}$ until total resuspension. To prepare variant $4,2.5 \% \mathrm{starch}$ and chitosan solutions (w/v) were mixed under agitation, and $400 \mu \mathrm{L}$ of $0.4 \%$ GA solution (w/v) were added. The mix was stirred for another 10 minutes at $800 \mathrm{rpm}$. All the solutions were poured into the molds, frozen at $-20{ }^{0} \mathrm{C}$ for 12 hours, and later thawed. This was repeated 3 times to complete 3 freeze-thaw cycles.

\section{Hydrogel characterization .}

Structure and composition of lyophilized hydrogels were studied by spectroscopy and microscopy techniques. Fourier transform infrared spectroscopy (FTIR) in a Nicolet Nexus FTIR (USA) was used to obtain hydrogel infrared spectra. The samples were analyzed at $25^{0} \mathrm{C}$, between $500-4000 \mathrm{~cm}^{-1}$. Using a JEOL JSM-6380 LV (USA) microscope, hydrogel tridimensional structure was observed by scanning electron microscopy (SEM), and its chemical composition was analyzed by energy-dispersive X-ray spectroscopy (EDS).

Hydrogels swelling in the presence of water was also studied. Each dry hydrogel sample was weighted before immersion in deionized water. At certain times during $24 \mathrm{~h}$, samples were weighted again and the swelling rate $(\mathrm{SR})$ was calculated using the formula: $\mathrm{SR}=(\mathrm{Ww}-\mathrm{Wd}) / \mathrm{Wd}$. Where $\mathrm{Ww}$ is wet weight, and $\mathrm{Wd}$ is dry weight of hydrogel samples. SR values were multiplied by 100 to obtain the percentual swelling rate of each hydrogel.

Nanoparticle synthesis .

CS-TPP nanoparticles (NPs) were synthesized by ionotropic gelation method as reported before (Canepa, 2017). Briefly: CS powder, $2 \mathrm{mg} / \mathrm{mL}$, was dissolved in acetic acid $2 \%$, under magnetic stirring. The solution was then filtered using $0.45 \mu \mathrm{m}$ membrane filters. NPs were obtained by dropwise addition of TPP at $1.5 \mathrm{mg} / \mathrm{mL}$ in water $(2 \mathrm{~mL}$ ), using a KDS 200 syringe infusion pump (USA), with $21 \mathrm{G} 11 / 2$ needle and speed $15 \mathrm{~mL} / \mathrm{h}$, under stirring. Resulting NPs suspension was stirred for 30 minutes and later stored at 4 ${ }^{0} \mathrm{C}$. Nanoparticle hydrodynamic diameter was analyzed by Dynamic light scattering (DLS) using a Malvern Zetasizer ZS-90 (UK). The size and shape were confirmed by scanning transmission electron microscopy (STEM) using a TESCAN MIRA 3 (Czech Republic).

Hydrogel interaction with nanoparticles .

In order to analyze hydrogel ability to absorb and retain nanoparticles, lyophilized hydrogel fragments, $6 \mathrm{~mm}$ in diameter and $8 \mathrm{~mm}$ long, were incubated with $50 \mathrm{mg}$ of CS-TPP NPs in phosphate buffer saline (PBS) $(3 \mathrm{~mL})$, for $3 \mathrm{~h}$ at $25{ }^{\circ} \mathrm{C}$. Then, the excess of PBS was removed, and NPs-loaded hydrogels were lyophilized once again. Interaction hydrogels/NPs was visualized by SEM.

\section{Cell viability experiment .}

Yellow tetrazolium (MTT) assay (van de Loosdrecht, 1994) was used to study the effect of hydrogels on the viability of Human epidermal type 2 cells (HEp-2). In brief, lyophilized hydrogels fragments of $3 \mathrm{~mm} \times 2$ $\mathrm{mm}$ and $2 \mathrm{~mm}$ thickness were incubated with HEp-2 cells in Dulbecco's Modified Eagle Medium (DMEM). Nontreated cells were used as viability control and the treatment with Triton X-100 (1\% v/v) was consider as $100 \%$ cytotoxicity control. The experiment was performed in a 96 well plate, incubated at $37{ }^{\circ} \mathrm{C}$ and $5 \% \mathrm{CO}_{2}$. After 24 hours, an equal volume of medium containing MTT $(2 \mathrm{mg} / \mathrm{mL})$ was added to each well. Incubation conditions were maintained for another 4 hours, before measuring absorbance at $570 \mathrm{~nm}$ in a Synergy HTX multi-mode microplate reader (Biotek, USA). 
In vivo studies .

The interaction of hydrogels with mice was studied in vivo in order to analyze its effects in tissue regeneration. Healthy animals (female CF-1, 30-40g, 4 weeks old) were separated in groups of 5 animals each. Skin lesions were produced as previously described (Choi, 2008), briefly, the animals were first anesthetized with ketamine/xylazine $(100 \mathrm{mg} / \mathrm{kg}$ and $5 \mathrm{mg} / \mathrm{kg}$, respectively) and the dorsal hairs were completely shaved. Then, the area was cleaned with povidone iodide and a hot metal ring (6 $\mathrm{mm}$ diameter) was used to induce a dorsal wound. The skin inside the circular mark was excised with scissors. Lyophilized hydrogel fragments (6 $\mathrm{mm}$ diameter, $2 \mathrm{~mm}$ thickness) were immediately placed on the wound, without any other bandages or scaffolds. The wound areas were measured and visually analyzed every 3 days, for 15 days. Animals without hydrogel applied were used as control of the experiment.In vivo experiments were accomplished following biosafety and bioethics principles. The Bioethics and Biosafety Committee, Biological Sciences School, Universidad de Concepción, approved the procedure.

\section{Results and Discussion .}

Synthesized hydrogels.

Physical method was elected for the synthesis of hydrogels. This method does not use chemical cross-linking agents, which can affect entrapped drugs or biomolecules (Gulrez, 2011). Having a starch-based structure, hydrogel polymerization was induced with heat $\left(121^{\circ} \mathrm{C}\right)$, followed by three freeze-thawing cycles. Three hydrogels were synthesized by this method: starch, starch-PVA and starch-CS. Different conditions (temperature, agitation and reagent quantities) were assayed until the semi-solid hydrogel structure was obtained. After the last freeze-thawing cycle, hydrogel samples were freeze-dried and analyzed by SEM.

A general first observation of microscopic images (Figure 1 ) confirms the obtaining of 3D networks in all the cases, but with several differences. Starch hydrogel has a cleaner structure, with well-defined channels (around $150 \mathrm{~nm}$ in diameter), which could capture therapeutic molecules or particles inside the matrix (Figure 1A-D ). Starch-PVA structure seems more rigid, with thinner channels and less network-like arrangement (Figure 1E-H ). While starch-CS has an interesting powder-like structure given by the existence of granules or beads that cover the channels and can be seen in the highest magnification images (Figure 1I-L ). Looking for a cleaner structure of starch-CS hydrogel, a second (chemical) synthesis reaction was performed, using GA as crosslinking agent. This procedure led to a clean 3D network (Figure 1M-P ), but less intricate and with wider channels than starch hydrogel.

Although variations in the procedure (reagents proportion, etc.) could determine different results in terms of network construction, it is interesting that microscopical structures of each of the three physical hydrogels suggests a way to apply it. Starch hydrogel, with clean channels and a complex structure seems optimum for drug or particle absorption and retention. Moreover, starch hydrophilic character will allow a fast load of water-dispersed particles or drugs, while the intricate hydrogel matrix could guarantee a relatively longlasting release.

Additionally, starch-PVA hydrogel, with a hard and rigid structure has a bone-like appearance. An exploration of its capabilities in stem-cell or bone transplants applications could give positive results, since PVA have been reported before for this kind of uses (Dashtdar, 2015; Peng, 2019). A biodegradable matrix with a solid character, could be an efficient scaffold for cell grow and multiplication, while fulfilling a structural function as well.

The presence of big particles in the starch-CS network and the fact that they appeared spontaneously, is a very interesting feature. First, this suggests some level of separation between the polymers used for hydrogel synthesis, that is an absence of physical combination. This was the logical reason which led us to the posterior application of a chemical method. Their composition and internal structure must be known in order to explore potential applications.

In this way, energy dispersive X-ray spectroscopy analysis of starch-CS hydrogel was performed, showing channel walls with a typical carbohydrate composition, while important levels of nitrogen were detected 
on the beads (Figure 2 ). Because of chitosan chemical structure, an aminated polysaccharide (Li, 2018), nitrogen in the beads indicates their chitosan composition. Instead, the absence of nitrogen in the walls of the channels indicates their starch composition. The internal structure of the beads was known through its interaction with the microscope electron beam. After some minutes of the beam hitting the beads, they collapsed, and its empty interior was observed (Figure 3 ). This confirmed a capsule-like structure, suggesting potential uses in the encapsulation of molecules (proteins, bioactive compounds), which need to be explored in the near future.

A possible mechanism that led to beads formation could be related to the interaction of chitosan chains with ions present in the solution, during hydrogel synthesis. This is a mechanism similar to the reaction involved inionotropic gelation (Pedroso-Santana, 2020), but this must be confirmed and studied in detail, in next works.

Fourier transform infrared analysis.

Chemical composition of the four hydrogels was analyzed by FTIR (Figure 4 ). As a result, some differences among each spectrum were observed and the main peaks are identified in Table $\mathbf{1}$. Three bands are consistently present in the four spectra: those related to C-O and C-O-C stretching: $1017-1022 \mathrm{~cm}^{-1}$ and $1079-1082 \mathrm{~cm}^{-1}$, respectively; and the one about $3300 \mathrm{~cm}^{-1}$, which is usually associated to the presence of -OH group (Henao, 2018). These bands have been previously observed in FTIR spectra of chitosan (Brugnerotto, 2001), PVA (Peng, 2019), and starch (Xu, 2005).

In the case of the band at $3300 \mathrm{~cm}^{-1}$, it is noticeably increased in the presence of chitosan. This effect can be a consequence of - $\mathrm{OH}$ groups reinforcement, but also could be related to the impact of $\mathrm{N}-\mathrm{H}$ stretching from chitosan molecules (Brugnerotto, 2001). This band shows differences between physically and chemically obtained hydrogels, suggesting a rearrangement of $-\mathrm{OH}$ and $-\mathrm{NH}_{2}$ groups. This is comprehensible, since the chemical mechanism for hydrogel synthesis involves the presence of new molecular bonds that do not exist in physical hydrogels (Gulrez, 2011). The stretching of $\mathrm{C}=\mathrm{O}$ bonds in amides showed signals at $1550 \mathrm{~cm}^{-1}$ and $1639 \mathrm{~cm}^{-1}$. Both were observable in the presence of chitosan, as a consequence of glucosamine units in chitosan molecules (Henao, 2018).

Interestingly, spectra from starch-PVA and starch hydrogel were quite different, suggesting strong interactions between both polymers that modified starch spectrum. The $-\mathrm{CH}_{2}$ symmetric stretching at $2359 \mathrm{~cm}^{-1}$ is an identifying feature of starch-PVA hydrogel, due to a PVA contribution (Ghabboun, 2012). These differences in the FTIR spectra are associated with the structural variations observed between both hydrogels and will surely be reflected on the properties of each one.

Swelling rate.

Hydrogel swelling rate characterizes its capacity to absorb aqueous fluids in an in vivo environment, which may be directly associated to the exchange of waste and nutrients. (Peng, 2019). Hydrogel samples were immersed in water and weighed to obtain the four SR curves (Figure 5 ). As a result, each hydrogel swelled a high volume of water during the first hour, independently of the polymeric composition. Then, water absorption slowed down reaching an equilibrium after 4 hours.

Starch-PVA hydrogel, consistent with its rigid appearance and smaller channel diameters, showed lower SR values. On the contrary, starch and starch-CS hydrogels presented similar properties, increasing its weight approximately five times. In the case of starch hydrogel, a tendency to lost water in time was observed; this could be related to a loss of structural integrity (Biduski, 2018). A higher swelling rate, which doubles the values of the other hydrogels, was observed for starch-CS-GA. This property is highly convenient for the absorption of water-dispersed drugs and particles; and also an appropriate feature for exudate incorporation in tissue engineering applications.

Effect of hydrogels on cell viability.

During the study of new drug formulations, polymeric scaffolds, or devices for biomedical applications, 
safety profiles are essential before their use in living cells and organisms (Rawal, 2019; Sudarsan, 2016). Using biocompatible materials is relevant for safe systems design, but it does not guarantee the absence of toxicity. Factors such as the presence of toxic reagents, even in small quantities, could induce some level of cytotoxicity (Ali, 2018). That is why, an in vitro viability assay is mandatory in this kind of studies.

HEp-2 cells viability in the presence of the four hydrogels was evaluated by MTT (Figure 6 ). The viability of the control cells (CC), incubated without hydrogels, was set as the $100 \%$ value. Incubation with Triton X-100 (1\% v/v), a non-ionic surfactant that induces cell lysis, was used as cytotoxicity control (CT).

Starch hydrogel resulted the safest material; while starch-chitosan, regardless of the method of synthesis, showed good viability percentages (higher than $80 \%$ ). In contrast, cells incubated with starch-PVA hydrogel were the most affected, presenting significantly lower values of cell viability. Considering that both polymers, starch and PVA, are biocompatible per se (Mansur, 2009; Perez, 2018), other factors related to hydrogel structure may be contributing to this result. The rigid starch-PVA structure and the lower water absorption, may influence the flow of substances through the hydrogel, originating areas of less access to nutrients in the plate. Particularly, by having less swelling, starch-PVA hydrogel remains drier and introduces a structural barrier to cell growth, leading to smaller values of cell viability.

The formation of rigid networks in PVA hydrogels indicates the existence of inter- and intra- polymer chain bonds; this decreases the number of exposed hydrophilic groups and reduces the swelling (Mansur, 2009; Tang, 2011). Starch-PVA hydrogels have been studied before, showing increased swelling with higher starch quantities (Hsie, 2013), and major (negative) effect on cell viability when PVA is a primary component of the hydrogel (Bernal-Ballen, 2019). According to these results, a possible way to decrease the negative effect on cell viability and to improve the swelling of our starch-PVA hydrogel could be an increase of starch quantity and/or a reduction of PVA content.

Synthesis of chitosan nanoparticles and incorporation into hydrogels.

Ionotropic gelation method has been used before to obtain polymeric nanoparticles able to encapsulate bioactive molecules. This type of encapsulation gives protection and control the release of the active principle. In addition, this method is relatively easy to perform in the laboratory (Pedroso-Santana, 2020). Polymeric nanoparticles can be loaded into a hydrogel, which could give a second barrier of protection against environmental agents, increasing bioavailability of encapsulated molecules even more. Also, the stable physical structure of hydrogels could serve as an anchoring mechanism in the sites of action, establishing a static position for releasing of bioactive molecules. Considering the aforementioned, ionotropic gelation was used to obtain chitosan nanoparticles, with sizes around $200 \mathrm{~nm}$ (Figure 7 ), and to study their interaction with hydrogels. Due to the previous results of low swelling and poor biocompatibility, starch-PVA hydrogel was excluded from next experiments.

The nanoparticles were synthesized in a reproducible way, showing an average particle diameter of $205 \mathrm{~nm}$ and a polydispersion index (PdI) of 0,198 (Figure 7A ). DLS results were confirmed by STEM, demonstrating the nanoparticle spherical shape and the high homogeneity of the population (Figure 7B-E ). Considering a potential use of these nanoparticles for drug delivery, two positive characteristics of the population can be mentioned: this size is effective for the interaction with cellular structures, such as membrane and cytoplasmic organelles (Pedroso-Santana, 2018), and the size homogeneity correlates with a homogeneous drug-loading capacity ( $\mathrm{Li}, 2015)$.

After characterization, the nanoparticles were centrifuged, and the pellet was dispersed in PBS. This aqueous dispersion containing agglomerates formed by the centrifugation step was used to submerge the hydrogels. Three hours later, the hydrogels were lyophilized and the physical interaction with the nanoparticles was visualized by SEM (Figure 8 ).

Starch hydrogel showed a higher quantity of nanoparticles adsorbed to the channel walls (Figure 8A-B ), in comparison to starch-CS (Figure 8C-D ). Considering that channel walls in both hydrogels are composed of starch, the lower number of nanoparticles in the second one could be due to the steric interference 
produced by the large chitosan beads. These beads introduce a barrier to the channel access (Figure 1K-L ), which would be interfering with the nanoparticle's adsorption onto hydrogel walls. This limitation was overcome in starch-CS-GA hydrogels. Obtaining a clean and smooth structure allowed the homogeneous nanoparticle's incorporation by the hydrogel (Figure 8E-F ). This hydrogel showed excellent interaction with the nanoparticles, based on their efficient incorporation and their regular distribution on the entire structure.

In order to use nanoparticles-loaded hydrogels in drug delivery applications, the system must retain a high quantity of nanoparticles and be able to release them in a controlled manner. In this way, the hydrogel structure guarantees the therapeutic effect of the drug at the site of action for a prolonged time. This will lead to a robust system for the administration of drugs with significant advantages such as: a controlled drug release and extended bioactivity (Wechsler, 2019). From the variants proposed in this work, Starch-CS-GA hydrogel showed the greatest response in terms of swelling rate, viability measured by MTT incorporation, and interaction with nanoparticles; therefore, this hydrogel was used for an in vivo experiment.

Starch-CS-GA hydrogel in vivo wound healing

experiment.

One way to apply this type of hydrogels for drug delivery and tissue engineering is to use them as wound dressing (Hamedi, 2018; Wechsler, 2019). Thereby, the hydrogel absorbs wound exudates, providing a balance of hydration and, also, using this aqueous environment to slowly release loaded drugs. In order to evaluate the general behavior of starch-CS-GA hydrogel in vivo, it was used in a mouse wound healing model (Figure 9 ). No nanoparticles were used in this experiment, only hydrogel effect was evaluated.

All wounds in control and treated animals had a $6 \mathrm{~mm}$ diameter on day 0 ; variations of this parameter were studied for 15 days (Figure 9A ). At the $3^{\text {rd }}$ day of the experiment, mice treated with hydrogels presented a dry wound, without redness or inflammation. In contrast, control animals showed inflamed, reddened, and exuding wounds. Because of this, an increase in the diameter of the wounds was observed in untreated animals, presenting a deterioration instead of the recovery observed in hydrogel-treated animals (Figure 9B ). A similar effect was observed on day 6 for both groups. But, around a week after the beginning of the experiment, the natural healing process was evident in control mice, which showed a reduction in wound diameters at day 9. However, wound healing effects were more evident in the treated animals. By day 12, all treated animals healed, while this process was not completed in untreated control mice.

The study revealed significative differences between both groups in wound healing during the complete experiment. The absence of inflammation and exudates in treated animals, along with faster healing, suggests the hydrogel beneficial effect. These results evidence the potentialities of starch-CS-GA hydrogel for wound dressing applications. Although no biologically active drugs or nanoparticles were used in thisin vivo study, the swelling properties and the efficient load of chitosan nanoparticles observed for this type of hydrogel, are encouraging evidences of its potential application in drug delivery.

\section{Conclusions .}

Based on its excellent properties, such as biodegradability, biocompatibility, and low cost, starch is a remarkably interesting choice for the development of polymer-based devices in biological applications. Its combination with other polymers could improve the mechanical and biological properties and facilitate the formation of functional hydrogels. PVA and chitosan interaction with starch led to the formation of hydrogels under certain conditions. Among the four synthesized hydrogels, the combination of starch and chitosan by chemical crosslinking showed the best results in terms of structure, swelling, and resulted safe in vitro and in vivo . The wound healing experiment in mice proved the potentialities of starch-CS-GA for tissue engineering applications; while its excellent interaction with chitosan nanoparticles evidenced a promissory behavior in the delivery of these nanoparticulate systems. The other three hydrogels presented relevant properties that justify next studies in order to fully exploit their potential.

\section{Conflict of interest .}


The authors have no conflict of interest to declare.

\section{References .}

Akhtar, M. F., Hanif, M., \& Ranjha, N. M. (2016). Methods of synthesis of hydrogels ... A review. Saudi Pharmaceutical Journal ,24 (5), 554-559. https://doi.org

/10.1016/j.jsps.2015.03.022

Ali, A., \& Ahmed, S. (2018). A review on chitosan and its nanocomposites in drug delivery. International Journal of Biological Macromolecules , 109 , 273-286. https://doi.org/10.1016/j.ijbiomac.2017.12.078.

Bernal-Ballen, A., Lopez-Garcia, J.-A., \& Ozaltin, K. (2019). (PVA/Chitosan/Fucoidan)-Ampicillin: A Bioartificial Polymeric Material with Combined Properties in Cell Regeneration and Potential Antibacterial Features. Polymers , 11 (8), 1325. https://doi.org/10.3390/polym11081325.

Biduski, B., Silva, W. M. F. da, Colussi, R., Halal, S. L. de M. E., Lim, L.-T., Dias, Á. R. G., \& Zavareze, E. da R. (2018a). Starch hydrogels: The influence of the amylose content and gelatinization method. International Journal of Biological Macromolecules ,113 , 443-449. https://doi.org/10.1016/j.ijbiomac.2018.02.144.

Brugnerotto, J., Lizardi, J., Goycoolea, F. M., Argüelles-Monal, W., Desbrières, J., \& Rinaudo, M. (2001). An infrared investigation in relation with chitin and chitosan characterization. Polymer ,42 (8), 3569-3580. https://doi.org/10.1016/S0032-3861(00)00713-8.

Bursali, E. A., Coskun, S., Kizil, M., \& Yurdakoc, M. (2011). Synthesis, characterization and in vitro antimicrobial activities of boron/starch/polyvinyl alcohol hydrogels. Carbohydrate Polymers ,83 (3), 1377-1383. https://doi.org/10.1016/j.carbpol.2010.09.056.

Cánepa, C., Imperiale, J. C., Berini, C. A., Lewicki, M., Sosnik, A., \& Biglione, M. M. (2017). Development of a Drug Delivery System Based on Chitosan Nanoparticles for Oral Administration of Interferona.Biomacromolecules , 18 (10), 3302-3309. https://doi.org/10.1021/acs.biomac.7b00959.

Choi, J. S., Leong, K. W., \& Yoo, H. S. (2008). In vivo wound healing of diabetic ulcers using electrospun nanofibers immobilized with human epidermal growth factor (EGF). Biomaterials , 29 (5), 587-596. https://doi.org/10.1016/j.biomaterials.2007.10.012.

Crescenzi, V., Francescangeli, A., Taglienti, A., Capitani, D., \& Mannina, L. (2003). Synthesis and Partial Characterization of Hydrogels Obtained via Glutaraldehyde Crosslinking of Acetylated Chitosan and of Hyaluronan Derivatives. Biomacromolecules , 4 (4), 1045-1054. https://doi.org/10.1021/bm0340669.

Dashtdar, H., Murali, M. R., Abbas, A. A., Suhaeb, A. M., Selvaratnam, L., Tay, L. X., \& Kamarul, T. (2015). PVA-chitosan composite hydrogel versus alginate beads as a potential mesenchymal stem cell carrier for the treatment of focal cartilage defects. Knee Surgery, Sports Traumatology, Arthroscopy , 23 (5), 1368-1377. https://doi.org/10.1007

/s00167-013-2723-5.

Fadouloglou, V. E., Kokkinidis, M., \& Glykos, N. M. (2008). Determination of protein oligomerization state: Two approaches based on glutaraldehyde crosslinking. Analytical Biochemistry ,373 (2), 404-406. https://doi.org/10.1016/j.ab.2007.10.027.

Fu, J., Yang, F., \& Guo, Z. (2018). The chitosan hydrogels: From structure to function. New Journal of Chemistry , 42 (21), 17162-17180. https://doi.org/10.1039/C8NJ03482F.

Ghabboun, J., Husseini, G. A., Faroun, M., Karmi, A., Hawash, Z., Hayek, I., \& Sowwan, M. (2012). Mapping of Embedded Functionalized Carbon Nanotubes in Poly(vinyl alcohol)/Nanotube Composite Using Electrostatic Force Microscopy. International Journal of Polymer Analysis and Characterization , 17 (4), 268-277. https://doi.org/10.1080

/1023666X.2012.658636. 
Gulrez, S. K., Al-Assaf, S., \& O, G. (2011). Hydrogels: Methods of Preparation, Characterisation and Applications. In A. Carpi (Ed.),Progress in Molecular and Environmental Bioengineering - From Analysis and Modeling to Technology Applications . InTech. https://doi.org/10.5772/24553.

Hamedi, H., Moradi, S., Hudson, S. M., \& Tonelli, A. E. (2018). Chitosan based hydrogels and their applications for drug delivery in wound dressings: A review. Carbohydrate Polymers , 199 , 445-460. https://doi.org/10.1016/j.carbpol.2018.06.114.

Henao, E., Delgado, E., Contreras, H., \& Quintana, G. (2018). Polyelectrolyte Complexation versus Ionotropic Gelation for Chitosan-Based Hydrogels with Carboxymethylcellulose, Carboxymethyl Starch, and Alginic Acid. International Journal of Chemical Engineering , 2018 , 1-12. https://doi.org/10.1155/2018/3137167.

Hou, Y., Chen, C., Liu, K., Tu, Y., Zhang, L., \& Li, Y. (2015). Preparation of PVA hydrogel with high-transparence and investigations of its transparent mechanism. RSC Advances , 5 (31), 24023-24030. https://doi.org/10.1039/C5RA01280E.

Hsieh, W.-C., \& Liau, J.-J. (2013). Cell culture and characterization of cross-linked poly(vinyl alcohol)-g-starch 3D scaffold for tissue engineering. Carbohydrate Polymers , 98 (1), 574-580. https://doi.org/10.1016/j.carbpol.2013.06.020.

Huang, N., Lin, J., Li, S., Deng, Y., Kong, S., Hong, P., Yang, P., Liao, M., \& Hu, Z. (2018). Preparation and evaluation of squid ink polysaccharide-chitosan as a wound-healing sponge. Materials Science and Engineering: C , 82 , 354-362. https://doi.org/10.1016/j.msec.2017.08.068.

Ismail, H., Irani, M., \& Ahmad, Z. (2013). Starch-Based Hydrogels: Present Status and Applications. International Journal of Polymeric Materials , 62 (7), 411-420. https://doi.org/10.1080/00914037.2012.719141.

Li, J., Cai, C., Li, J., Li, J., Li, J., Sun, T., Wang, L., Wu, H., \& Yu, G. (2018). Chitosan-Based Nanomaterials for Drug Delivery.Molecules , 23 (10), 2661. https://doi.org/10.3390/molecules23102661.

Li, Q., Liu, C.-G., \& Yu, Y. (2015). Separation of monodisperse alginate nanoparticles and effect of particle size on transport of vitamin E. Carbohydrate Polymers , 124 , 274-279. https://doi.org/10.1016/j.carbpol.2015.02.007.

Liu, Y., Geever, L. M., Kennedy, J. E., Higginbotham, C. L., Cahill, P. A., \& McGuinness, G. B. (2010). Thermal behavior and mechanical properties of physically crosslinked PVA/Gelatin hydrogels. Journal of the Mechanical Behavior of Biomedical Materials ,3 (2), 203-209. https://doi.org/10.1016/j.jmbbm.2009.07.001.

López-Córdoba, A., Estevez-Areco, S., \& Goyanes, S. (2019). Potato starch-based biocomposites with enhanced thermal, mechanical and barrier properties comprising water-resistant electrospun poly (vinyl alcohol) fibers and yerba mate extract. Carbohydrate Polymers , 215 , 377-387. https://doi.org/10.1016/j.carbpol.2019.03.105.

Mansur, H. S., de S. Costa, E., Mansur, A. A. P., \& Barbosa-Stancioli, E. F. (2009). Cytocompatibility evaluation in cell-culture systems of chemically crosslinked chitosan/PVA hydrogels. Materials Science and Engineering: C , 29 (5), 1574-1583. https://doi.org/10.1016/j.msec.2008.12.012.

Ou, A., \& Bo, I. (2017). Chitosan Hydrogels and their Glutaraldehyde-Crosslinked Counterparts as Potential Drug Release and Tissue Engineering Systems - Synthesis, Characterization, Swelling Kinetics and Mechanism. Journal of Physical Chemistry \&3 Biophysics , 07 (03). https://doi.org/10.4172/2161-0398.1000256.

Pedroso-Santana, S., Fleitas-Salazar, N., Sarabia-Sainz, A., Silva-Campa, E., Angulo-Molina, A., Pedroza-Montero, M., \& Riera, R. (2018). Nanodiamonds and gold nanoparticles to obtain a hybrid nanostructure with potential applications in biomedicine.Nanotechnology, 29 (43), 5101-5107. https://doi.org/10.1088/1361-6528/aadad1. 
Pedroso-Santana, S., \& Fleitas-Salazar, N. (2020). Ionotropic gelation method in the synthesis of nanoparticles/microparticles for biomedical purposes. Polymer International , 69 , 443-447. https://doi.org/10.1002/pi.5970.

Peng, L., Zhou, Y., Lu, W., Zhu, W., Li, Y., Chen, K., Zhang, G., Xu, J., Deng, Z., \& Wang, D. (2019). Characterization of a novel polyvinyl alcohol/chitosan porous hydrogel combined with bone marrow mesenchymal stem cells and its application in articular cartilage repair. BMC Musculoskeletal Disorders , 20 (1), 257. https://doi.org/10.1186/s12891-019-2644-7.

Perez, J. J., Francois, N. J., Maroniche, G. A., Borrajo, M. P., Pereyra, M. A., \& Creus, C. M. (2018). A novel, green, low-cost chitosan-starch hydrogel as potential delivery system for plant growth-promoting bacteria. Carbohydrate Polymers , 202 , 409-417. https://doi.org/10.1016/j.carbpol.2018.07.084.

Rawal, M., Singh, A., \& Amiji, M. M. (2019). Quality-by-Design Concepts to Improve Nanotechnology-Based Drug Development. Pharmaceutical Research , 36 (11), 153. https://doi.org/10.1007/s11095-019-2692-6.

Sudarsan, S., Franklin, D. S., Sakthivel, M., \& Guhanathan, S. (2016). Non toxic, antibacterial, biodegradable hydrogels with $\mathrm{pH}$-stimuli sensitivity: Investigation of swelling parameters. Carbohydrate Polymers , 148 , 206-215. https://doi.org/10.1016/j.carbpol.2016.04.060.

Tang, X., \& Alavi, S. (2011). Recent advances in starch, polyvinyl alcohol based polymer blends, nanocomposites and their biodegradability.Carbohydrate Polymers , 85 (1), 7-16. https://doi.org/10.1016/j.carbpol.2011.01.030.

Timaeva, O., Pashkin, I., Mulakov, S., Kuzmicheva, G., Konarev, P., Terekhova, R., Sadovskaya, N., Czakkel, O., \& Prevost, S. (2020). Synthesis and physico-chemical properties of poly(N-vinyl pyrrolidone)-based hydrogels with titania nanoparticles. Journal of Materials Science, 55 (7), 3005-3021. https://doi.org/10.1007/s10853-019-04230-z.

van de Loosdrecht, A. A., Beelen, R. H. J., Ossenkoppele, G. J., Broekhoven, M. G., \& Langenhuijsen, M. M. A. C. (1994). A tetrazolium-based colorimetric MTT assay to quantitate human monocyte mediated cytotoxicity against leukemic cells from cell lines and patients with acute myeloid leukemia. Journal of Immunological Methods , 174 (1-2), 311-320. https://doi.org/10.1016/0022-1759(94)90034-5.

Vrsanska, M., Voberkova, S., Jimenez, A. M., Strmiska, V., \& Adam, V. (2018). Preparation and optimisation of cross-linked enzyme aggregates using native isolate white rot fungi Trametes versicolor andFomes fomentarius for the decolourisation of synthetic dyes.International Journal of Environmental Research and Public Health , 15 , 23. https://doi.org/10.3390/ijerph15010023.

Wechsler, M. E., Stephenson, R. E., Murphy, A. C., Oldenkamp, H. F., Singh, A., \& Peppas, N. A. (2019). Engineered microscale hydrogels for drug delivery, cell therapy, and sequencing. Biomedical Microdevices , 21 (2), 31. https://doi.org/10.1007/s10544-019-0358-0.

Xu, Y. X., Kim, K. M., Hanna, M. A., \& Nag, D. (2005). Chitosan-starch composite film: Preparation and characterization. Industrial Crops and Products , 21 (2), 185-192. https://doi.org/10.1016/j.indcrop.2004.03.002.

Table .

\begin{tabular}{|c|c|c|c|c|c|}
\hline Peak $\left(\mathrm{cm}^{-1}\right)$ & $\begin{array}{l}\text { Possible } \\
\text { assignment }\end{array}$ & Starch & Starch-PVA & Starch-CS & $\begin{array}{l}\text { Starch-CS- } \\
\text { GA }\end{array}$ \\
\hline $3274-3420$ & $\begin{array}{l}\text { O-H stretching } \\
\text { (Henao, 2018) } \\
\text { N-H stretching } \\
\text { (Brugnerotto, } \\
2001 \text { ) }\end{array}$ & yes & yes & yes (increased) & yes (increased) \\
\hline
\end{tabular}




\begin{tabular}{|c|c|c|c|c|c|}
\hline Peak $\left(\mathrm{cm}^{-1}\right)$ & $\begin{array}{l}\text { Possible } \\
\text { assignment }\end{array}$ & Starch & Starch-PVA & Starch-CS & $\begin{array}{l}\text { Starch-CS- } \\
\text { GA }\end{array}$ \\
\hline 2359 & $\begin{array}{l}-\mathrm{CH}_{2-} \\
\text { symmetric } \\
\text { stretching } \\
\text { (Ghabboun, } \\
\text { 2012) }\end{array}$ & no & yes & no & yes (reduced) \\
\hline 1079-1082 & $\begin{array}{l}\text { C-O-C stretching } \\
\text { (Henao, 2018) }\end{array}$ & yes & yes & yes & yes \\
\hline 1639 & $\begin{array}{l}\mathrm{C}=\mathrm{O} \text { bond of } \\
\text { amides } \\
(\text { Henao, 2018) }\end{array}$ & no & no & no & yes \\
\hline $1551-1556$ & $\begin{array}{l}\mathrm{C}=\mathrm{O} \text { bond of } \\
\text { amides } \\
(\text { Henao, 2018) }\end{array}$ & no & no & yes & yes \\
\hline 1409-1410 & $\begin{array}{l}\text { C-H bonds } \\
(\text { Henao, 2018) }\end{array}$ & no & no & yes & yes \\
\hline 1017-1022 & $\begin{array}{l}\text { C-O stretching } \\
\text { (Henao, 2018) }\end{array}$ & yes & yes & yes & yes \\
\hline
\end{tabular}

Table 1. Principal bands observed in Fourier transform infrared spectra and their relation with each hydrogel.

Figure legends .

Figure 1 . Scanning electron microscopy images of the four hydrogels. A-D : starch. E-H : starchpolyvinylalcohol.I-L : starch-chitosan. M-P : starch-chitosan-glutaraldehyde.

Figure 2 . Microscopic images and energy-dispersive X-ray spectroscopy analysis of starch-chitosan hydrogel. A : channel surface. B : small bead surface. $\mathbf{C}$ : big bead surface.

Figure 3 . Chitosan beads capsule-like structure observed by scanning electron microscopy. A : compact structure before continuous exposure to the electron beam. B : empty interior uncovered after exposure to the electron beam (red arrows).

Figure 4 . Fourier transform infrared spectra of the hydrogels. A : starch. B : starch-polyvinylalcohol. C : starch-chitosan. D : starch-chitosan-glutaraldehyde.

Figure 5. Swelling rate of each hydrogel during $24 \mathrm{~h}$.

Figure 6 . Cytotoxicity results of hydrogels in HEp-2 cells (average of three independent experiments. * Differences detected by ANOVA analysis with $\mathrm{p}<0.05)$.

Figure 7 . Chitosan nanoparticles characterization in terms of size and shape. A : Dynamic light scattering results of three nanoparticle batches. B-E : Scanning transmission electron microscopy images of the nanoparticles.

Figure 8 . Scanning electron microscopy images of hydrogel interaction with chitosan nanoparticles. A-B : starch hydrogel.C-D : starch-chitosan hydrogel. E-F : starch-chitosan-glutaraldehyde. Red arrows point to the nanoparticles.

Figure 9 . Starch-chitosan-glutaraldehyde hydrogel effect in a mouse wound healing model. A : Experiment scheme. B : Comparison of hydrogel effect on wound diameter against control without hydrogel (* Differences detected by ANOVA analysis with $\mathrm{p}<0.05)$. 


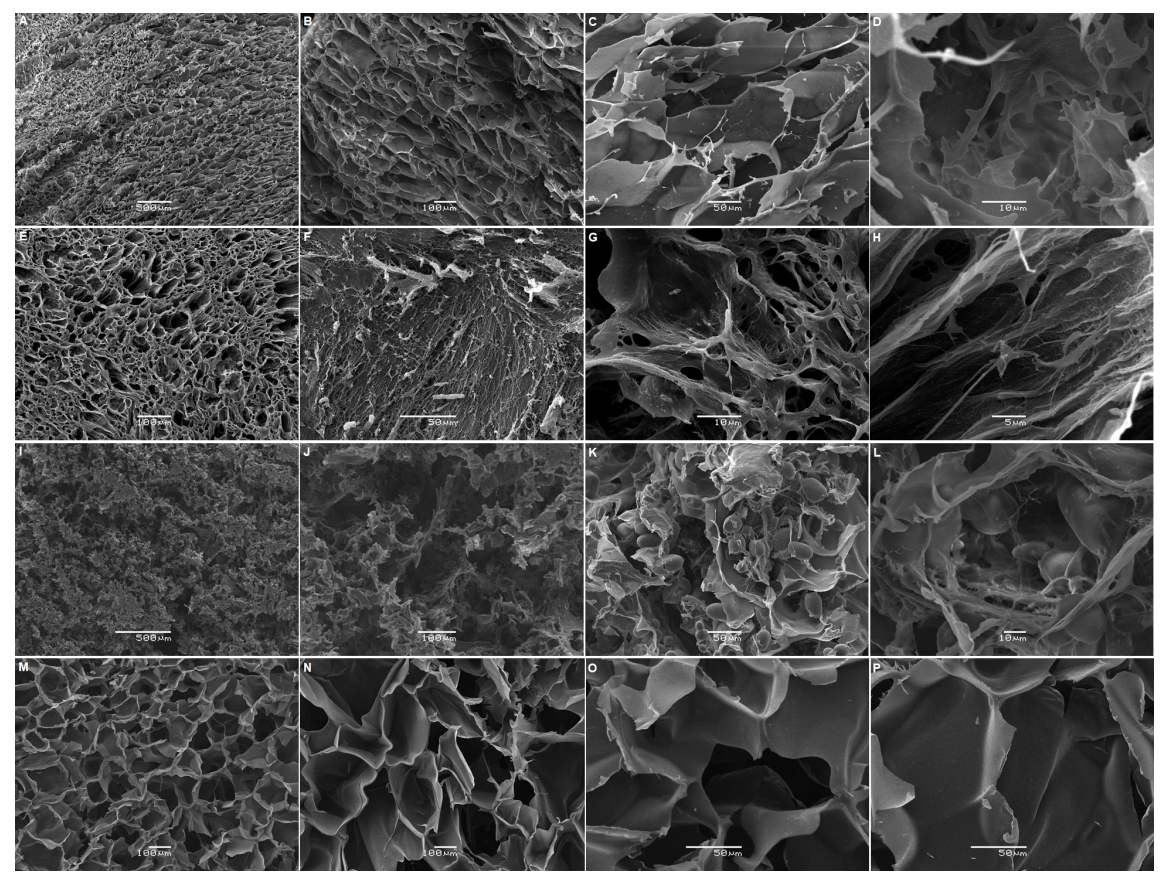




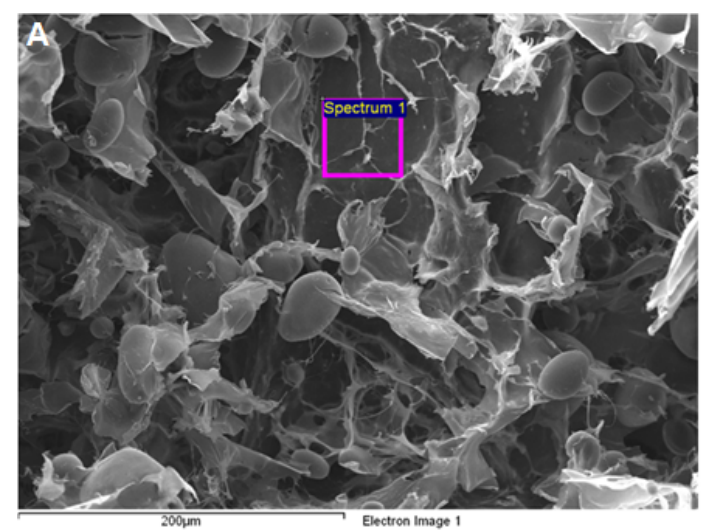

\begin{tabular}{l|ll}
\hline Element & Weight\% & Atomic\% \\
C K & 59.57 & 66.24 \\
OK & 40.43 & 33.76 \\
& & \\
Totals & 100.00 & \\
\hline
\end{tabular}

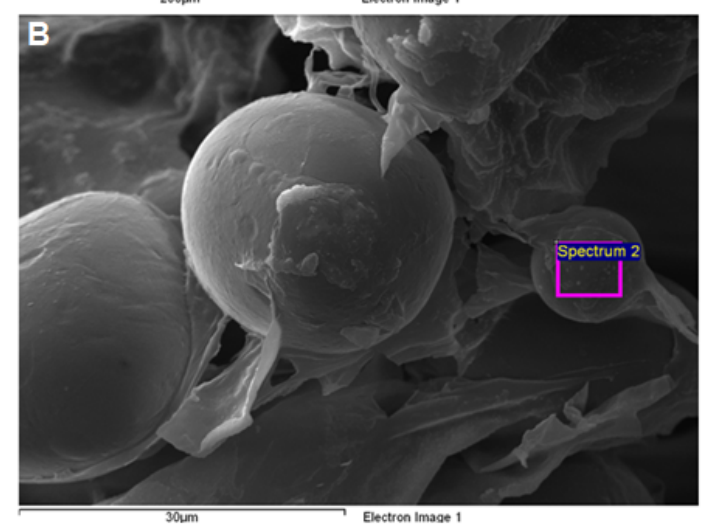

\begin{tabular}{l|ll}
\hline Element & Weight\% & Atomic\% \\
CR & 45.60 & 53.1 \\
NK & 15.79 & 13.75 \\
OK & 34.80 & 3.38 \\
NaK & 2.70 & 1.64 \\
CI K & 3.11 & 1.22 \\
Totals & 100.00 & \\
\hline
\end{tabular}

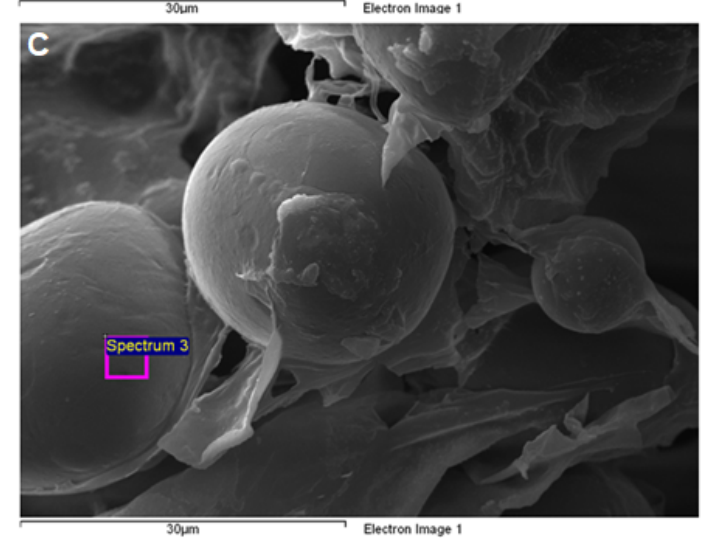

\begin{tabular}{l|ll}
\hline Element & Weight\% & Atomic\% \\
$\mathrm{CK}$ & 56.29 & 62.99 \\
$\mathrm{~N} \mathrm{~K}$ & 13.01 & 12.49 \\
$\mathrm{OK}$ & 27.44 & 23.05 \\
$\mathrm{NaK}$ & 1.16 & 0.68 \\
$\mathrm{Cl} \mathrm{K}$ & 2.10 & 0.80 \\
& & \\
Totals & 100.00 & \\
\hline
\end{tabular}
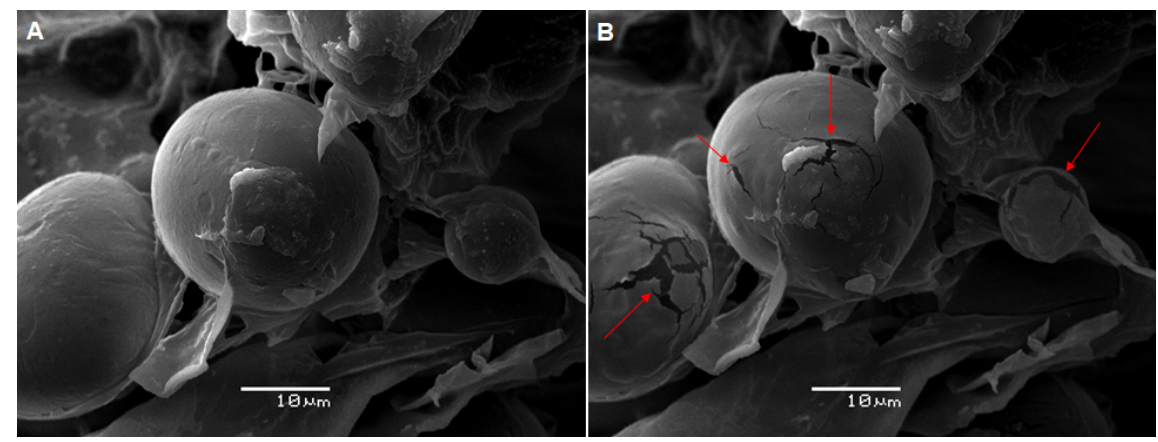


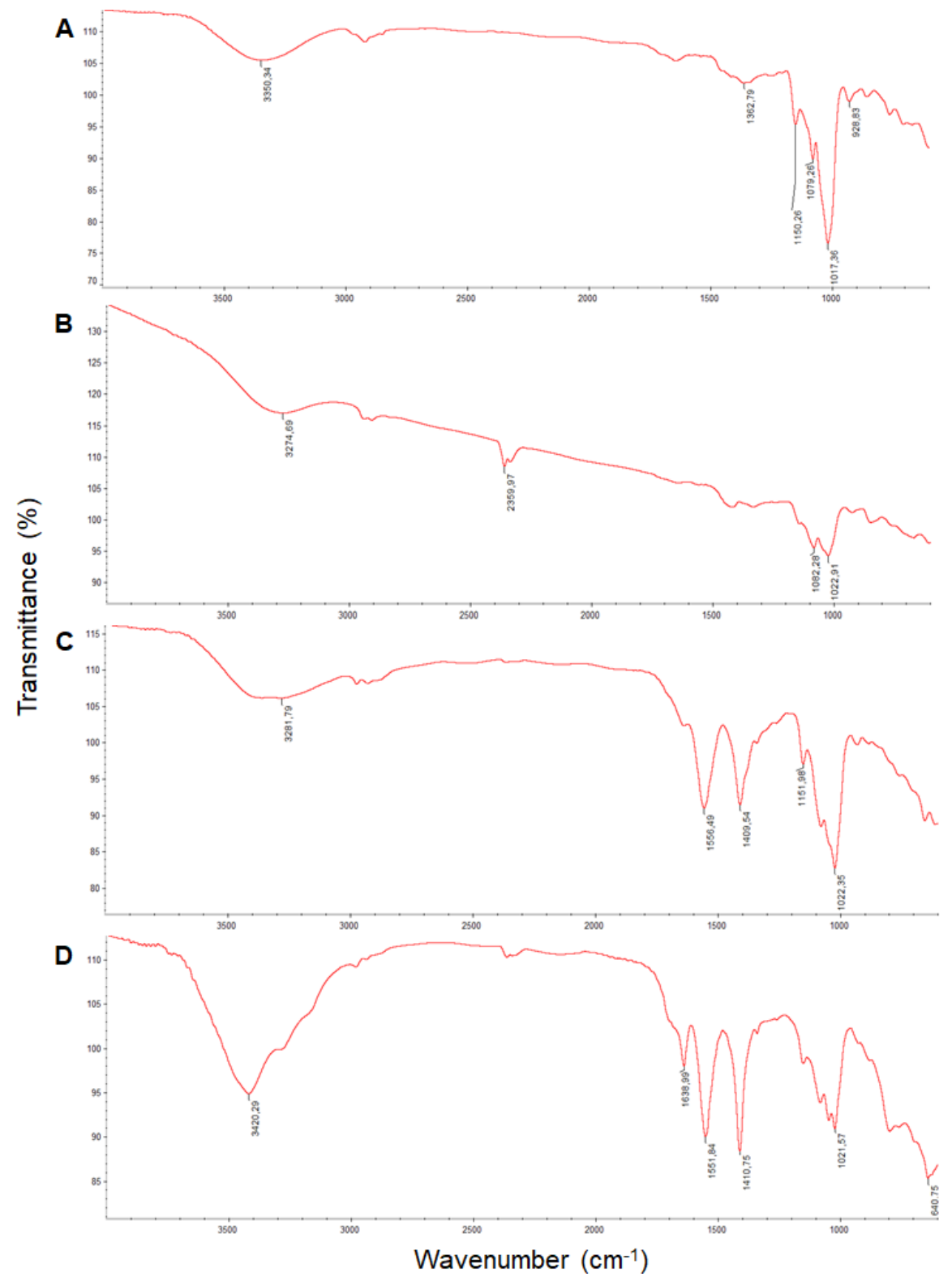



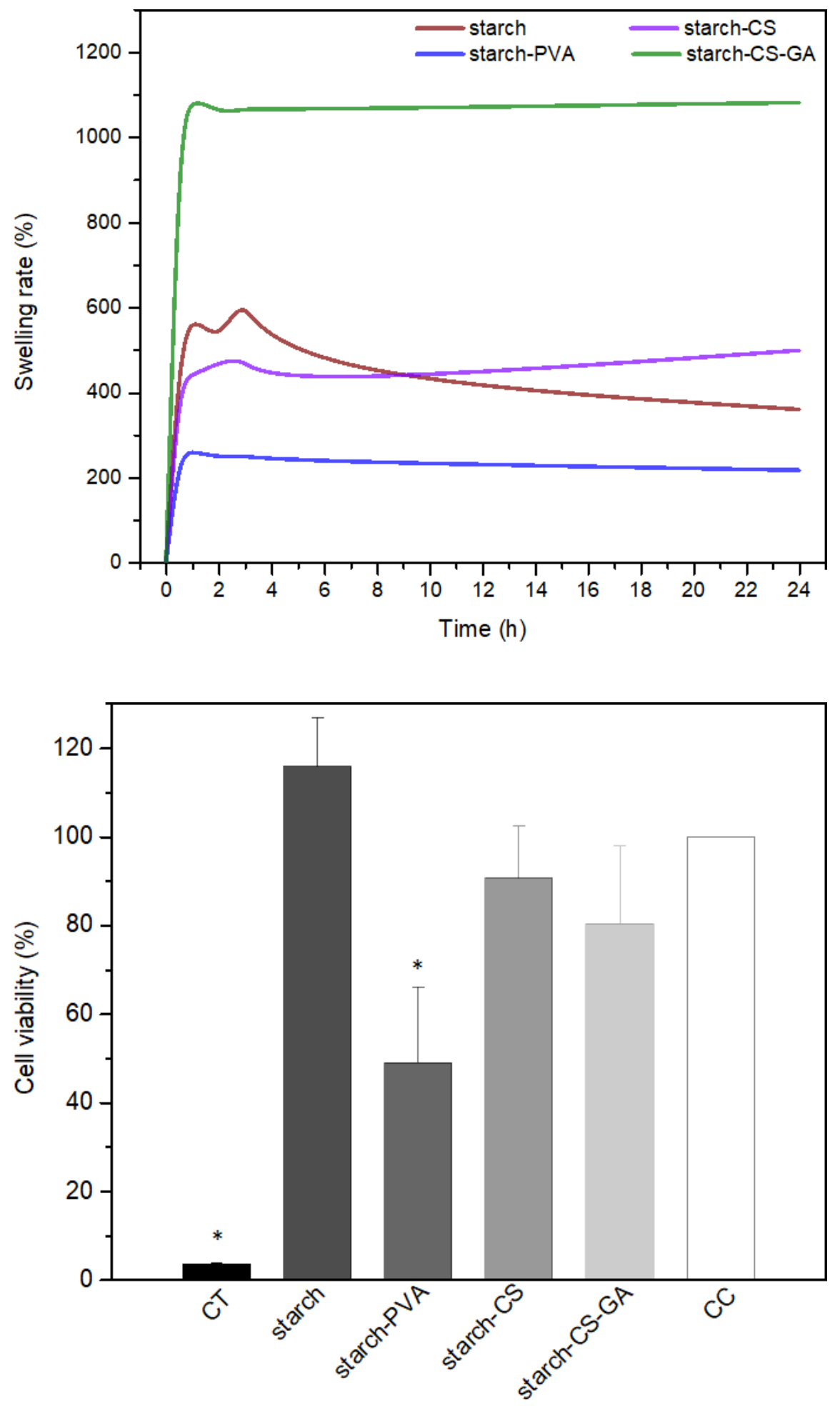
A
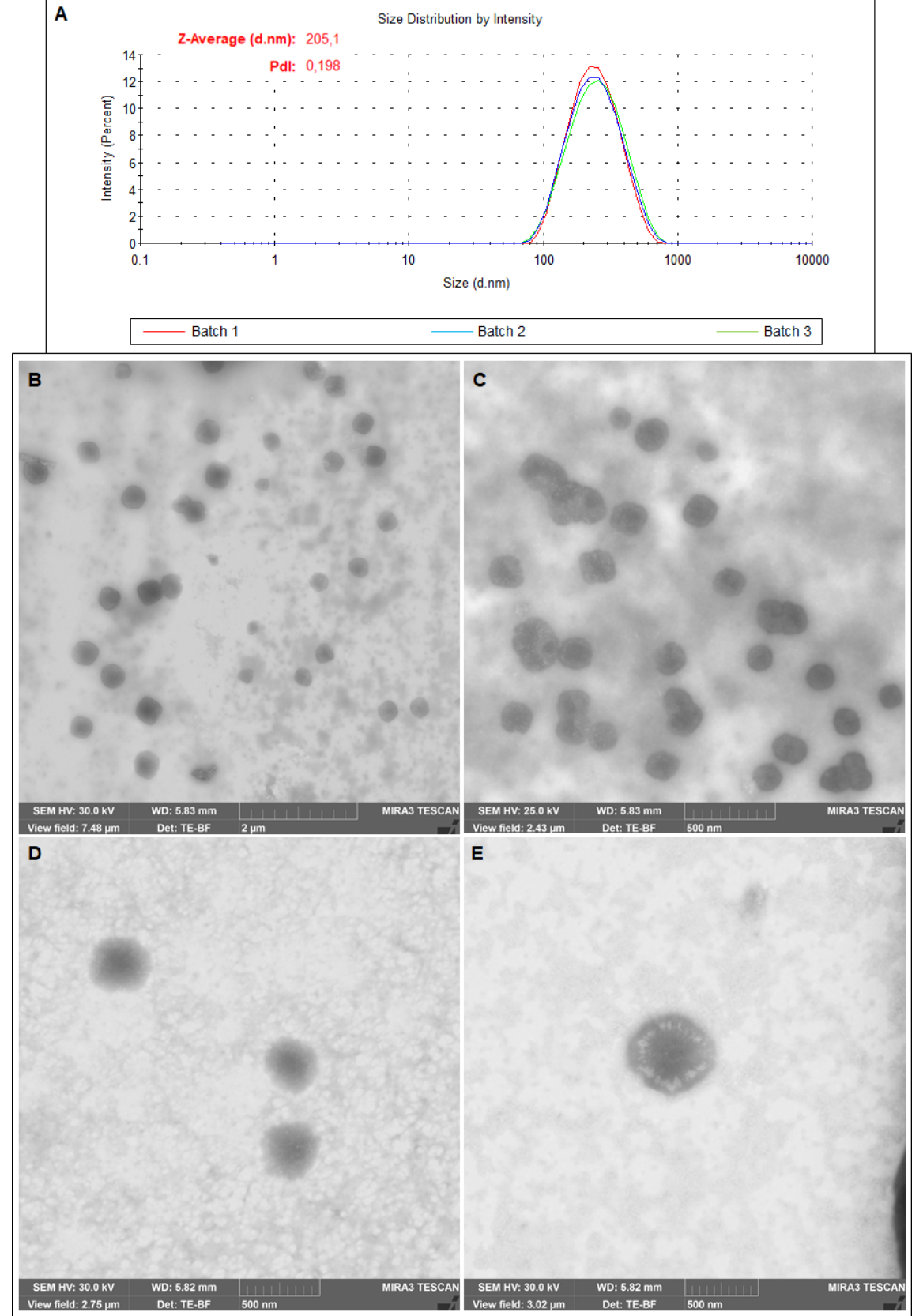


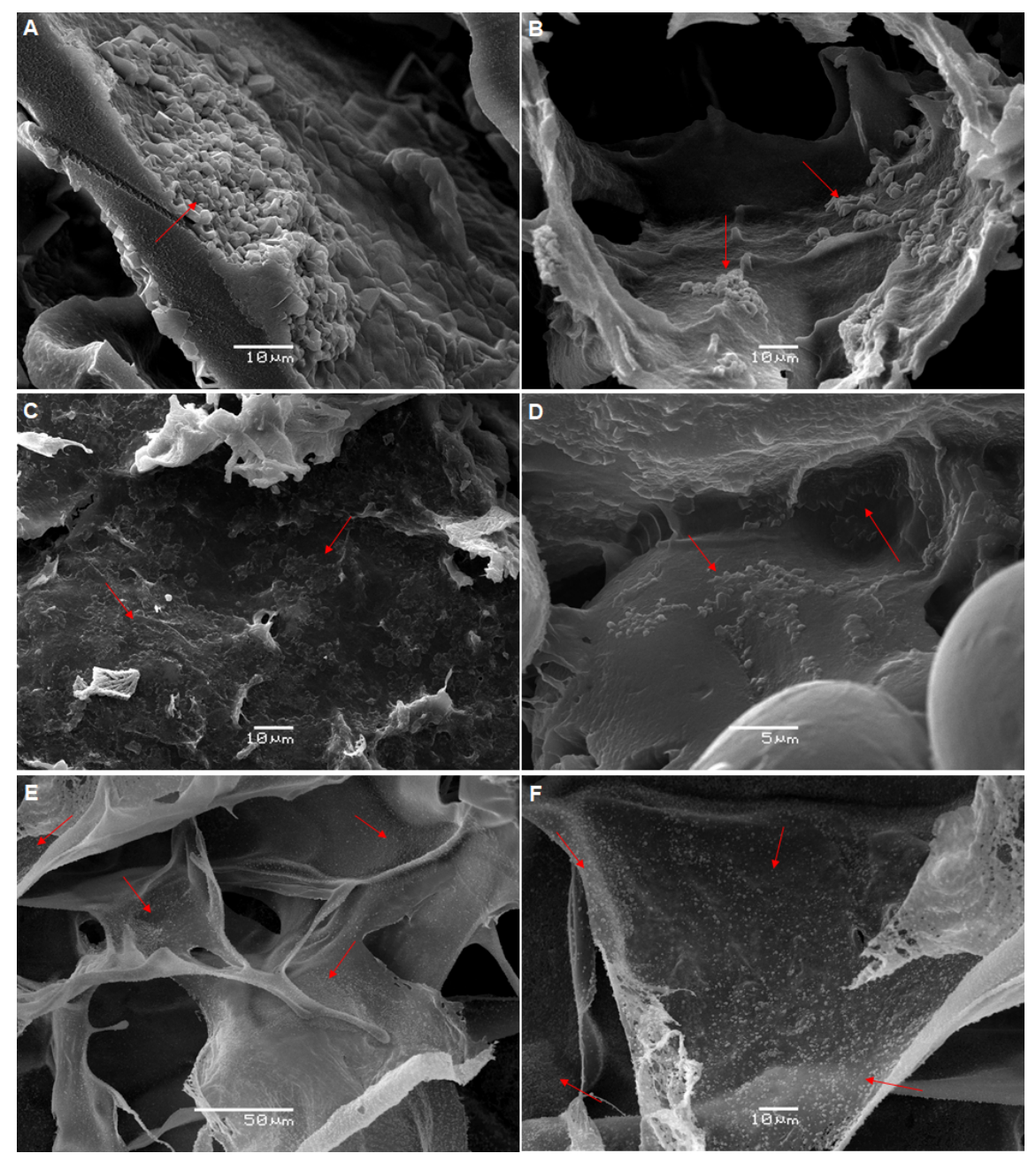




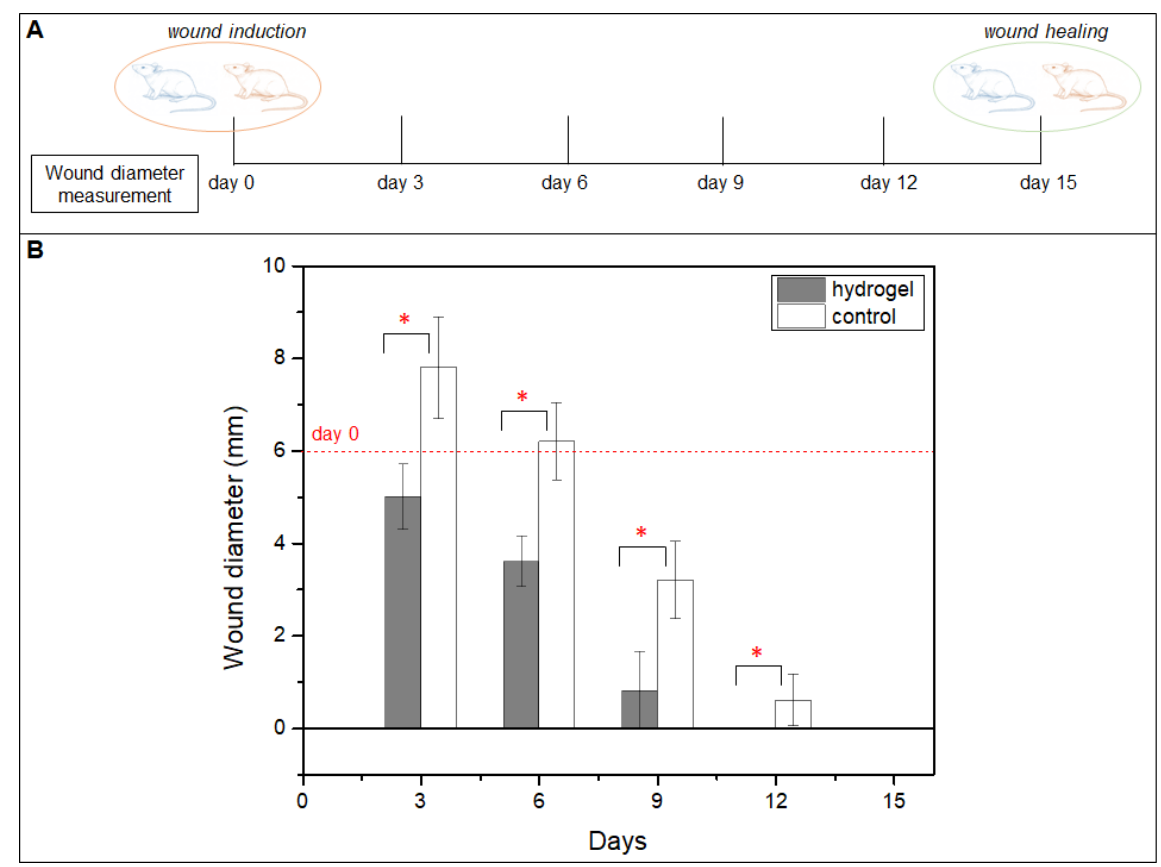

